\title{
Synthesis of High-Quality Multilayer Hexagonal Boron Nitride Films on Au Foils for Ultrahigh Rejection Ratio Solar-Blind Photodetection
}

Biying Tan, Huihui Yang*, Yunxia Hu, Feng Gao, Lifeng Wang, Mingjin Dai, Shichao Zhang, Huiming Shang, Hongyu Chen*, PingAn $\mathrm{Hu}^{*}$

Key Laboratory of Micro-systems and Micro-structures Manufacturing of Ministry of Education, Harbin Institute of Technology, Harbin 150001, P. R. China.

*E-mail: hupa@hit.edu.cn; chenhy@hit.edu.cn; yhh@hit.edu.cn

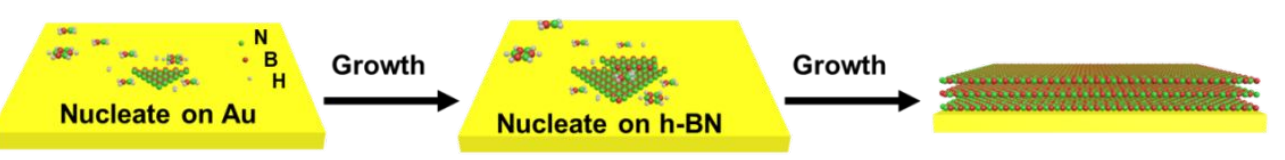

Figure S1. Schematic diagram of the CVD synthesis of multilayer h-BN films on Au foils. 


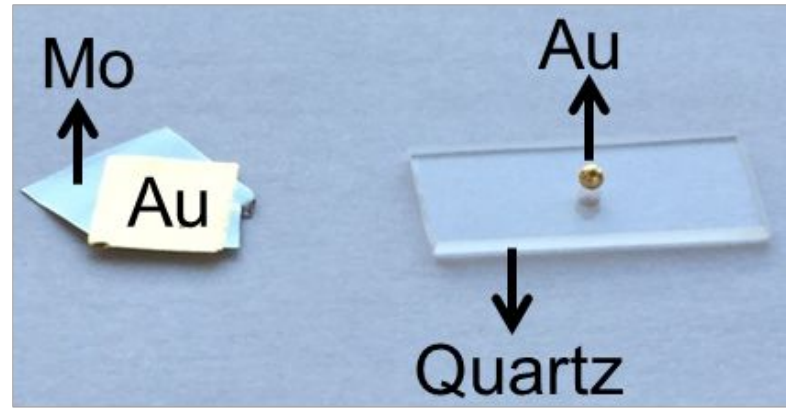

Figure S2. Au foil on Mo supporting substrate after h-BN synthesis, and Au foil on quartz supporting substrate after h-BN synthesis.

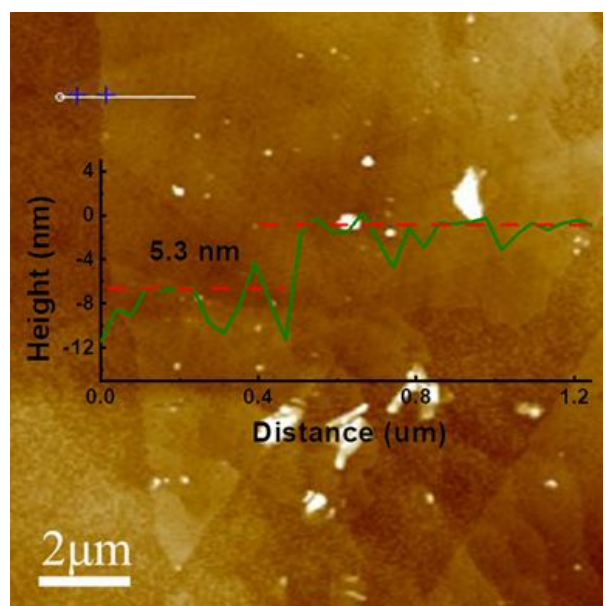

Figure S3. AFM image of multilayer h-BN flakes with a thickness of about $5 \mathrm{~nm}$ on Au foils after 5-min growth.

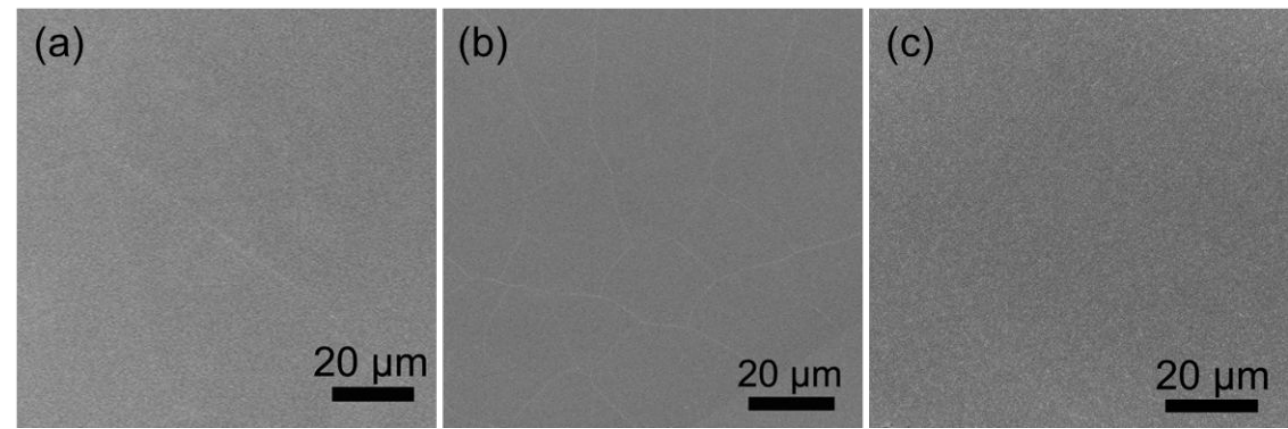

Figure S4. SEM images of Au foil before and after h-BN synthesis. (a) Au foil before growth of h-BN film. (b) Au foil after growth of h-BN film for 10 mins, (c) Au foil after growth of h-BN film for 20 mins. 


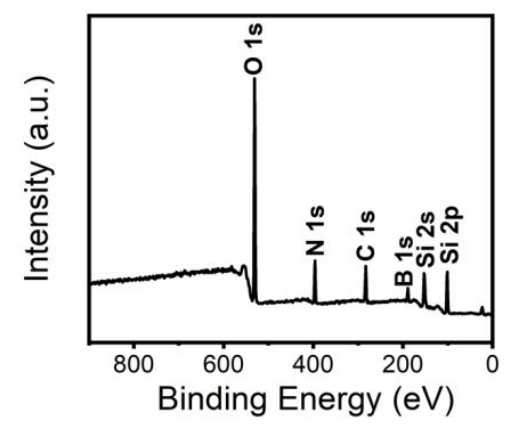

Figure S5. Survey XPS spectrum of multilayer h-BN films transferred on $300 \mathrm{~nm} \mathrm{SiO}_{2} / \mathrm{Si}$ substrates.

(a)

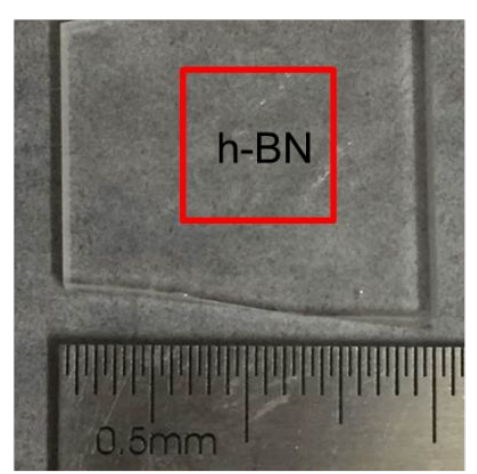

(b)

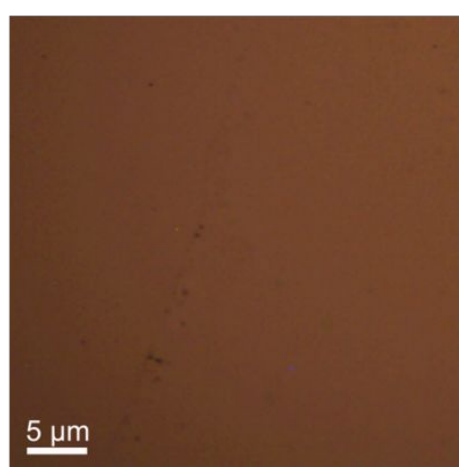

Figure S6. (a) Photograph and (b) OM images of multilayer h-BN film transferred onto quartz substrates for optical absorption measurement.

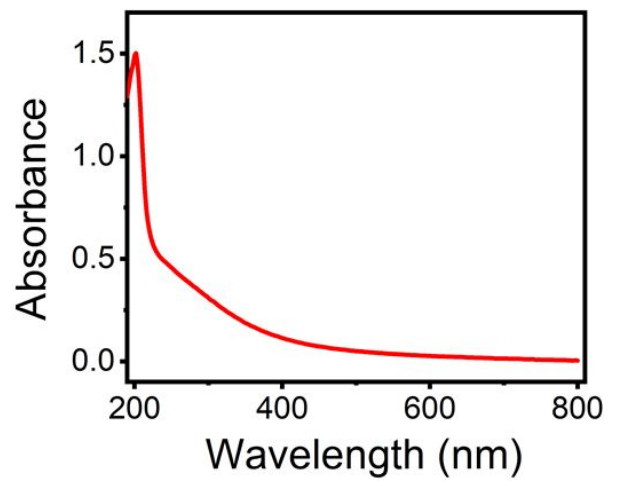

Figure S7. Optical absorption spectrum of h-BN films with the thickness of $\sim 11 \mathrm{~nm}$. 

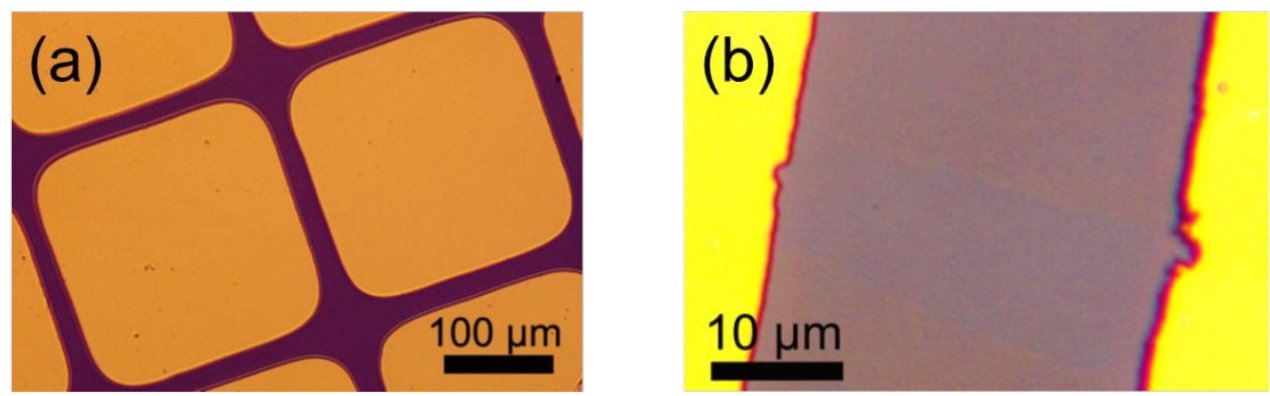

Figure S8. OM images of the solar-blind h-BN photodetector device. (a) Low-resolution OM image of the device. (b) High-resolution OM image of the device, the yellow material on either side is the electrode, the violet material is the $\mathrm{SiO}_{2} / \mathrm{Si}$ substrate, the relatively dark violet, ribbon-like material in the middle is active material h-BN. The size of electrodes is $\sim 230 \times 230 \mu \mathrm{m}^{2}$, the size of active material of $\mathrm{h}-\mathrm{BN}$ is about $30 \times 10 \mu \mathrm{m}^{2}$.

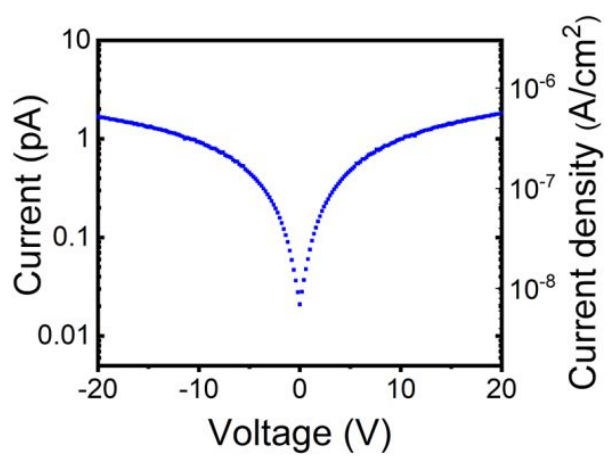

Figure S9. Current and current density of the solar-blind h-BN photodetector device under 250 $\mathrm{nm}$ light illumination at $20 \mathrm{~V}$.

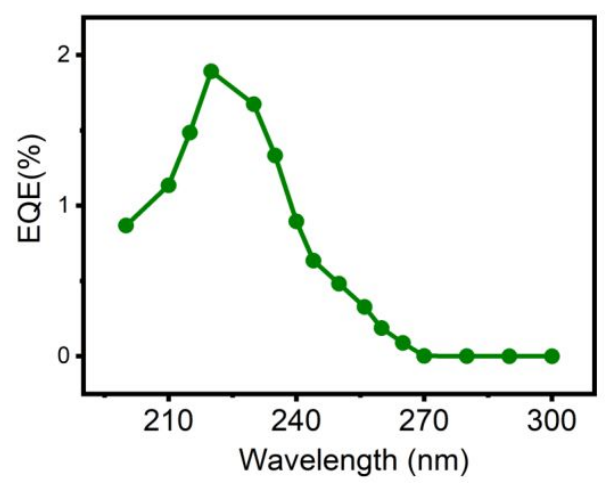

Figure S10. EQE of the solar-blind h-BN photodetector measured under different wavelengths irradiation at $20 \mathrm{~V}$. 

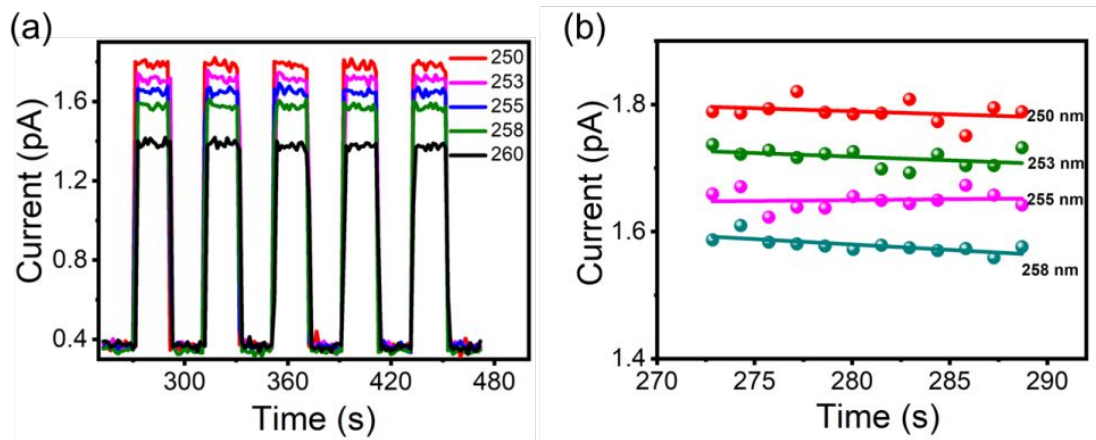

Figure S11. (a) $I-t$ curves for slightly different wavelengths differentiation. (b) High-resolution $I-$ $t$ curves by linear fitting for slightly different wavelengths differentiation. 